\title{
Current differences in referral patterns for pulmonary endarterectomy in the UK
}

\author{
M. Toshner*, $f$, J. Suntharalingam ${ }^{\#, f}$, K. Goldsmith ${ }^{*}$, A. Niggebrugge ${ }^{+}$, \\ J. Pepke-Zaba*, N.W. Morrell ${ }^{\S}$, S. Tsui*, J. Flowers ${ }^{+}$and D. Jenkins*
}

ABSTRACT: Pulmonary endarterectomy (PEA) surgery is the treatment of choice in surgically accessible chronic thromboembolic pulmonary hypertension and is potentially curative. The UK is served by seven specialist pulmonary hypertension centres and, consequently, there are regions which do not have a specialist unit. Since 2000, Papworth Hospital (Papworth Everard, UK) has been the sole PEA provider for the UK, offering the opportunity to study the national incidence of operable disease and give potential insight into factors that might affect geographical distribution within the UK.

All 262 UK residents who underwent PEA surgery between April 2000 and May 2006 were included in the present study. The age-adjusted cumulative referral rates were compared between regions to test for uniformity.

Overall, observed rates differed significantly from expected, with evidence of significant nonuniformity across the UK. The highest rates were observed in proximity to the nationally designated specialist centres and in particular in East Anglia and the West Midlands, nearest Papworth. These two regions differed by $>\mathbf{2} \times$ SD from the national mean rate.

The present study demonstrates wide geographical variation in the number of patients referred for pulmonary endarterectomy surgery. This suggests that there may be patients who are not presently being offered this potentially curative option.

\section{KEYWORDS: Pulmonary hypertension, thromboendarterectomy}

$\mathbf{T}$ here is emerging data to suggest that, across the spectrum of diseases causing pulmonary hypertension, there is widespread underdiagnosis [1, 2]. In this context, recent studies of chronic thromboembolic pulmonary hypertension (CTEPH) estimated rates of up to $3.8 \%$ of acute pulmonary embolic events [3], much higher than previously thought. Untreated CTEPH leads to right ventricular dysfunction and death [4]. It has been suggested that the rarity of this condition may be compounded by under-recognition $[4,5]$; this is of particular relevance as pulmonary endarterectomy (PEA) surgery, the treatment of choice in surgically accessible disease, can be curative. When good surgical clearance of disease is obtained, there is a fall in pulmonary vascular resistance with near-normalisation of cardiopulmonary function and an excellent long-term prognosis [6].

The UK population is served by seven specialist centres that are designated by the National Specialist Commissioning Advisory Group. This means there are regions that do not have a direct specialist centre. In addition, Papworth Hospital
(Papworth Everard, UK), one of the seven centres, has developed a PEA programme that has accumulated the world's third-largest surgical series since its inception in 1997. Since April 2000, this programme has been funded as the sole provider of PEA surgical services to the UK. This unique situation offers an excellent opportunity to study UK national referral patterns for the disease. By studying the geographical distribution of cases suitable for PEA surgery, the aim of the present study was to give some insight into the level of awareness of CTEPH within the UK, and suggest factors that may affect this.

\section{MATERIALS AND METHODS \\ Study subjects}

All UK residents who underwent PEA surgery between April 2000 and May 2006 were analysed. Patients with CTEPH are currently considered for surgery when sufficient surgically accessible (i.e. proximal) disease can be demonstrated on imaging (at least two methods out of computed tomography pulmonary angiography, catheterdirected pulmonary angiography and magnetic resonance pulmonary angiography) in the presence of both significant symptoms and deranged
AFFILIATIONS

*Papworth Hospital NHS Trust,

Papworth Everard,

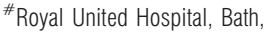

"Medical Research Council (MRC)

Biostatistics Unit,

+Eastern Region Public Health

Observatory (ERPHO), Institute of

Public Health,

§University of Cambridge School of Clinical Medicine, Addenbrooke's Hospital, Cambridge, UK.

${ }^{f}$ Both authors contributed equally to this article.

\section{CORRESPONDENCE}

J. Suntharalingam

Royal United Hospital

Bath

UK

Fax: 441480364998

E-mail: jaysunth@btopenworld.com

Received:

January 152008

Accepted after revision:

April 072008

SUPPORT STATEMENT

M. Toshner is funded by a grant from the British Heart Foundation.

STATEMENT OF INTEREST

None declared.

European Respiratory Journa

Print ISSN 0903-1936

Online ISSN 1399-3003 


\section{TABLE 1 Baseline demographics of study subjects}

\section{Age yrs}

Male \%

WHO functional class II/III/IV \%

6-min walking test $\mathrm{m}$

Right atrial pressure $\mathrm{mmHg}$

Mean pulmonary arterial pressure $\mathrm{mmHg}$

Cardiac index $\mathrm{L} \cdot \mathrm{min}^{-1} \cdot \mathrm{m}^{-2}$

Total pulmonary resistance dynes $\cdot \mathrm{s}^{-1} \cdot \mathrm{cm}^{-5}$

Data are presented as mean $\pm \mathrm{SD}$, unless otherwise stated. WHO: World Health Organization

\begin{tabular}{ll} 
TABLE 2 & $\begin{array}{l}\text { Age-adjusted cumulative rates of surgical cases } \\
\text { per 1,000,000 population, by region }\end{array}$ \\
Region & Cumulative rate $\mathbf{( 9 5 \%} \mathbf{~ C l )}$ \\
\hline East of England & $7.6(5.50-10.24)$ \\
East Midlands & $6.9(4.65-9.84)$ \\
North East & $4.9(2.58-8.30)$ \\
London & $4.5(2.98-6.54)$ \\
Yorkshire and Humber & $3.9(2.41-6.10)$ \\
Scotland & $3.4(2.03-5.41)$ \\
Wales & $4.1(2.11-7.20)$ \\
West Midlands & $4.3(2.73-6.46)$ \\
North West & $3.8(2.48-5.57)$ \\
South West & $3.3(1.98-5.30)$ \\
South East & $3.6(2.38-5.11)$ \\
Northern Ireland & $1.2(0.13-4.34)$ \\
\hline &
\end{tabular}

resting pulmonary haemodynamics (mean pulmonary artery pressure $>25 \mathrm{mmHg}$ and pulmonary vascular resistance $>3$ Wood units).

\section{Study design and methods}

In order to compare geographical variation in referral patterns, mid-2004 population estimates were obtained from national registries in the UK. Direct age-adjusted cumulative rates were calculated for patients undergoing surgery from each region using standard techniques [7]. Subjects were allocated to regions through the use of Geographical Information Systems mapping software (MapInfo Professional V8.5; Pitney Bowes, New York, NY, USA), which relies upon home postcodes. Therefore, patients referred to a specialist centre and subsequently to Papworth were still accredited to the area of original referral. Subjects from England were assigned to one of nine regions that corresponded to Strategic Health Authorities. The remaining subjects were allocated to Scotland, Wales or Northern Ireland, as appropriate.

\section{Analysis}

The null hypothesis that cumulative rates were uniform across the UK was tested using the Chi-squared test, comparing observed versus expected rates. Regional rates were also

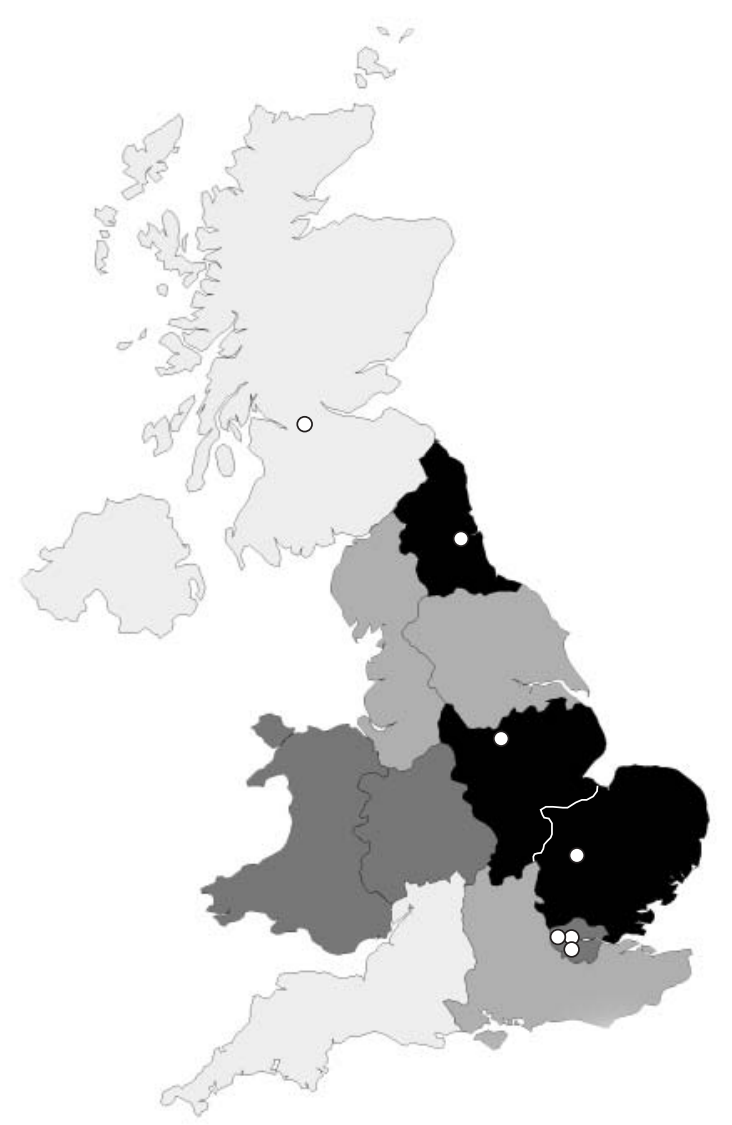

FIGURE 1. Geographic origin of referrals to the centre in Papworth (Cambridge, UK) for pulmonary endarterectomy. $\bigcirc$ : location of specialist centres. Referral rates per 1,000,000 population, with three regions within each band: $4.9-$ $7.6(\square) ; 4.1-4.9(\square) ; 3.6-4.1(\square) ; 1.2-3.6(\square)$

compared with a funnel plot analysis to identify regions with higher or lower than expected rates, allowing for variations in sample size. All data was anonymised.

\section{RESULTS}

In total, 262 subjects were included in the present study. Baseline demographics are shown in table 1. Regional observed rates differed significantly from expected, with evidence of significant nonuniformity across the UK $(p=0.0029$, Chi-squared test).

Cumulative rates for each of the 12 regions are demonstrated in table 2 and are also shown illustratively in figure 1. The highest rates were observed in close proximity to the nationally designated specialist centres and in particular in East Anglia and the West Midlands, the two regions nearest Papworth. These regions differed by $>2 \times \mathrm{SD}$ (fig. 2 ). All of the regions with a specialist centre had higher rates of referral than those without a specialist centre, with the exception of Scotland.

\section{DISCUSSION}

The present authors propose that the geographical pattern indicates a variable awareness of the availability and success of 


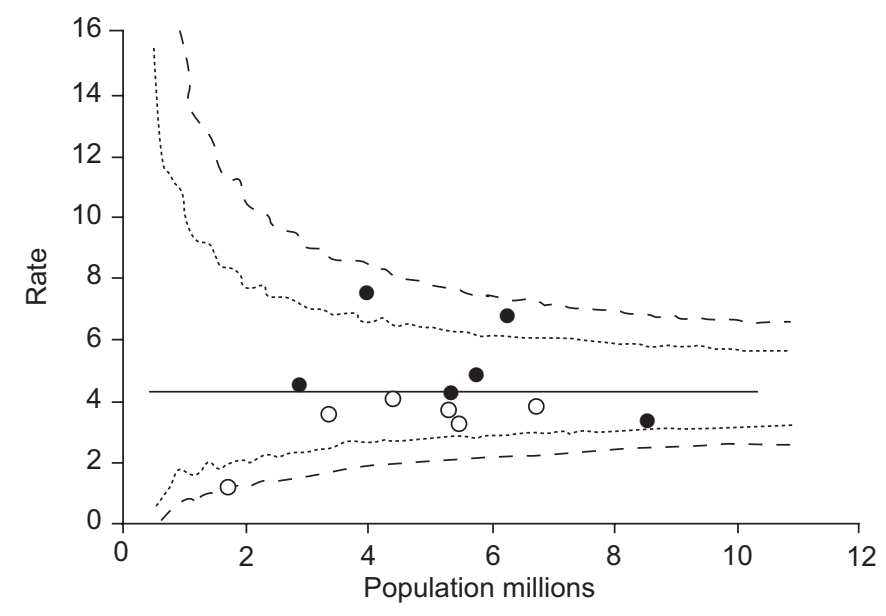

FIGURE 2. Funnel plot of age-adjusted cumulative rates of surgical cases per $1,000,000$ population, by region. $\mathbf{0}$ : region containing specialist referral centres; $\bigcirc$ : region with no specialist referral centres. London (UK) contains three specialist referral centres. —-: average rate; …...... upper and lower $2 \times$ SD limit; -------: upper and lower $3 \times$ SD limit.

PEA and that there are patients with life-threatening CTEPH who are not presently being offered this potentially curative option. Rates of surgical disease were higher in areas with a specialist centre, with the exception of Scotland. This demonstrates that a local high profile of specialists interested in the disease is the most likely factor driving increased referral rates. The Scottish centre is not a local referral service in the same manner as the English centres, as it covers a catchment area comparable with the rest of the centres combined and is a national referral service. The present authors feel this does not detract from the case for direct local interest being an important factor.

It is well documented that patients with CTEPH are often diagnosed late in their clinical course and that acute pulmonary embolus, the presumed aetiological trigger for $\mathrm{CTEPH}$, is itself often missed [8]. Traditionally, estimates from retrospective data have suggested that CTEPH complicated just $0.1 \%$ of patients who survive an acute embolic event, and that a lengthy "honeymoon" period often exists between initial presentation and subsequent development of symptomatic CTEPH [9]. More recently, this has been challenged with prospective data suggesting that the incidence of CTEPH following an acute event lies closer to $1-3.8 \%$ and can occur within the first 2 yrs [3, 5, 10]. Whether straightforward increased education in the wider community of professionals who deal with acute emboli is a sufficient strategy, or whether a longitudinal screening approach should be taken, is a debate that is crucially dependent on widespread acceptance of the benefits of the operation. Without acceptance that there is a sufficiently successful treatment, the idea of screening becomes less relevant. Although PEA is in its second decade, it is still a significant undertaking. The traditionally high mortality rates initially associated with the procedure have, however, been steadily coming down and more recent reports, such as in San Diego (USA), the world's largest centre, show mortality rates down to $4.4 \%$ [4]. It is possible, therefore, that the issue may not be underdiagnosis per se, but a lack of awareness of the improvements made in PEA.

There are alternative explanations for this uneven distribution of cases. One possibility is true geographical variability in disease prevalence. However, examination of the known risk factors for CTEPH (splenectomy, ventriculo-atrial shunts, antiphospholipid antibody syndrome and chronic inflammatory states [11]) do not suggest any genetic or environmental reason to support this. Alternatively, the distribution may be related to regional variations in the management of acute pulmonary embolism. It is possible, but less likely, that aggressive management of acute embolic disease in some regions, e.g. with thrombolytic therapy, may have led to a lower prevalence. Conversely, a lower prevalence rate could also result if patients with acute disease were less aggressively managed, leading to fewer at-risk survivors. Finally, the skewed distribution could be attributed to inequity of access to the PEA service as a result of patient selection bias, distance issues or socio-economic factors. By analysing only those patients who were operated on and not all referrals, the current authors have attempted to minimise the bias favouring centres with lower thresholds for referral and ensured that, as much as possible, only truly operable disease was examined.

The demonstration of variable referral rates for pulmonary endarterectomy surgery within the UK adds increasing evidence to the contention that chronic thromboembolic pulmonary hypertension is an underdiagnosed condition. Regardless of the explanation for this variability, it suggests that there are potentially operable patients being overlooked. As patients with pulmonary embolus are often seen by a broad variety of specialties, including respiratory, cardiology and haematology professionals, it is important that more is done across the medical spectrum to draw attention to this situation and increase awareness of the availability of this potentially curative option.

\section{ACKNOWLEDGEMENTS}

The authors are indebted to all the staff at Papworth Hospital (Papworth Everard, UK) who helped develop the pulmonary endarterectomy programme in Papworth Hospital over the last two decades.

\section{REFERENCES}

1 Peacock AJ, Murphy NF, McMurray JJ, Caballero L, Stewart S. An epidemiological study of pulmonary arterial hypertension. Eur Respir J 2007; 30: 104-109.

2 Humbert M. The burden of pulmonary hypertension. Eur Respir J 2007; 30: 1-2.

3 Pengo V, Lensing AW, Prins MH, et al. Incidence of chronic thromboembolic pulmonary hypertension after pulmonary embolism. N Engl J Med 2004; 350: 2257-2264.

4 Lang IM. Chronic thromboembolic pulmonary hypertension - not so rare after all. N Engl J Med 2004; 350: 22362238.

5 Tapson VF, Humbert M. Incidence and prevalence of chronic thromboembolic pulmonary hypertension: from acute to chronic pulmonary embolism. Proc Am Thorac Soc 2006; 3: 564-567. 
6 Jamieson SW, Kapelanski DP, Sakakibara N, et al. Pulmonary endarterectomy: experience and lessons learned in 1,500 cases. Ann Thorac Surg 2003; 76: 1457-1462.

7 National Cancer Institute. Surveillance Epidemiology and End Results. Calculating Age-adjusted Rates. http:// seer.cancer.gov/seerstat/tutorials/aarates/definition.html Date last accessed: November 2007.

8 Ryu JH, Olson EJ, Pellikka PA. Clinical recognition of pulmonary embolism: problem of unrecognized and asymptomatic cases. Mayo Clin Proc 1998; 73: 873-879.
9 Fedullo PF, Auger WR, Channick RN, Moser KM, Jamieson SW. Chronic thromboembolic pulmonary hypertension. Clin Chest Med 1995; 16: 353-374.

10 Miniati M, Monti S, Bottai M, et al. Survival and restoration of pulmonary perfusion in a long-term follow-up of patients after acute pulmonary embolism. Medicine (Baltimore) 2006; 85: 253-262.

11 Lang I, Kerr K. Risk factors for chronic thromboembolic pulmonary hypertension. Proc Am Thorac Soc 2006; 3: 568-570. 